\title{
HHV-6-derived microRNAs in the serum of DIHS/DRESS patients
}

Kazuya Miyashita, Fumi Miyagawa, Yuki Nakamura, Rie Onmori, Hiroaki Azukizawa, Hideo Asada Department of Dermatology, Nara Medical University School of Medicine

\section{Introduction}

Drug-induced hypersensitivity syndrome/ Drug reaction with eosinophilia and systemic ymptoms (DIHS/DRESS)

- A particular type of severe cutaneous adverse drug reaction

- Associated with the reactivation of human herpesvirus 6 (HHV-6)

\section{MicroRNA (miRNA)}

- Non-protein-coding small RNA in the size range of 19-25 nucleotides - Important roles in biological processes (immune response, cell and apoptosis)

- Many members of the herpesvirus family have their own miRNAs, which may regulate key viral genes

- In vitro study shows that hhv6b-miR-Ro6-1, 2, 3 and 4

(HHV6-miR-1, 2, 3 and 4) are detected in HHV-6 infected ce

- The precise role of these miRNAs in regulating HHV-6

latency and reactivation is largely unknown

Hhv6b-miR-Ro6-2

Hhv6b-miR-Ro6-3

Hhv6b-miR-Ro6-1

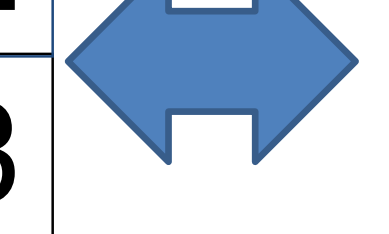

(expressed as early as 6h following infection) immediate early (IE) or early (E)

(Detected 2 days later than Hhv6b-miR-2,3)

Hhv6b-miR-Ro6-4 development, cell differentiation, organogenesis, growth control

Method

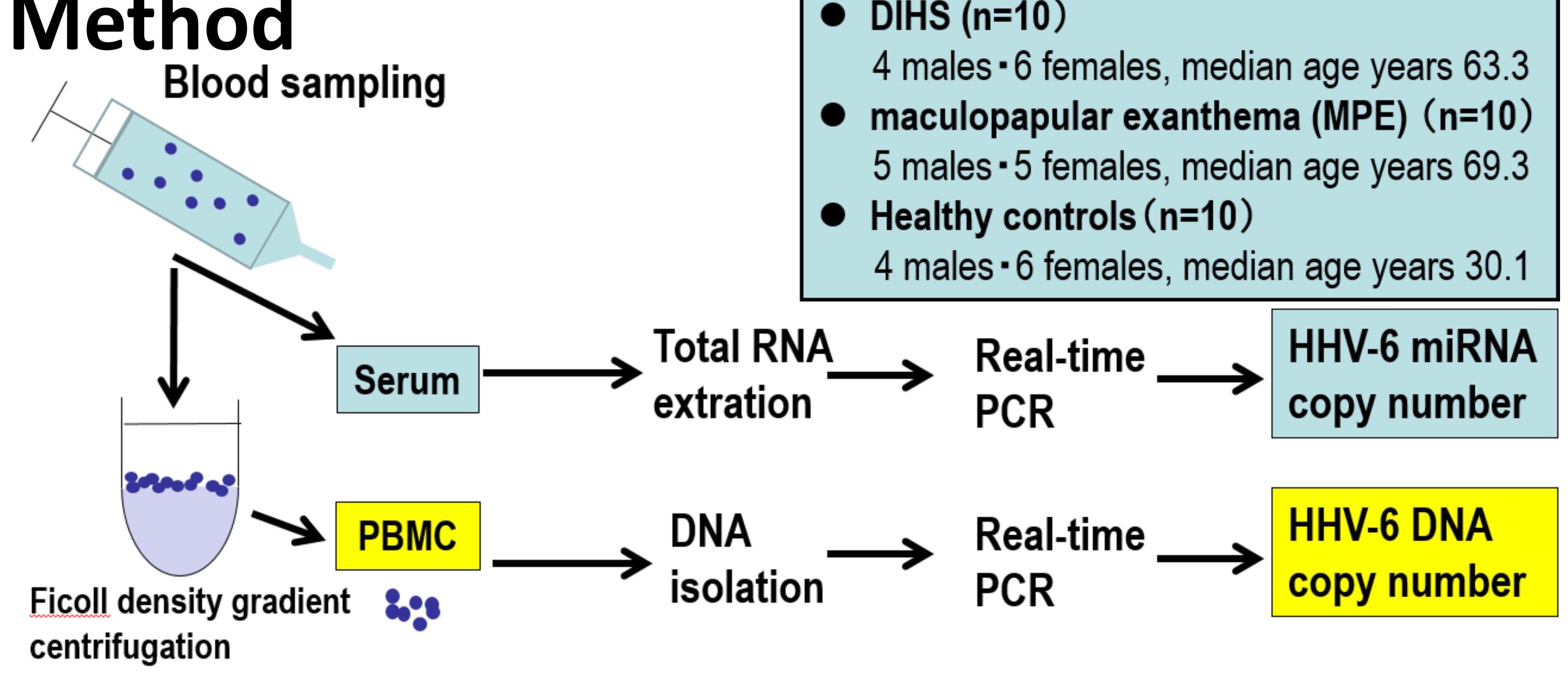

A summary of clinical and laboratory features of patients with DIHS/DRESS

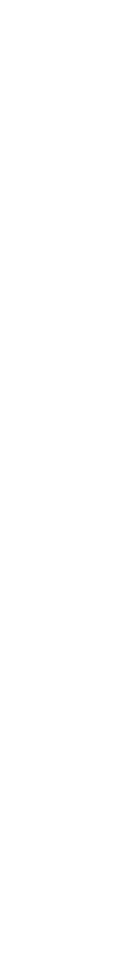

\section{Purpose of study}

- To investigate expression level of four HHV-6 miRNAs in serum of patients with DIHS/DRESS at the acute stage.

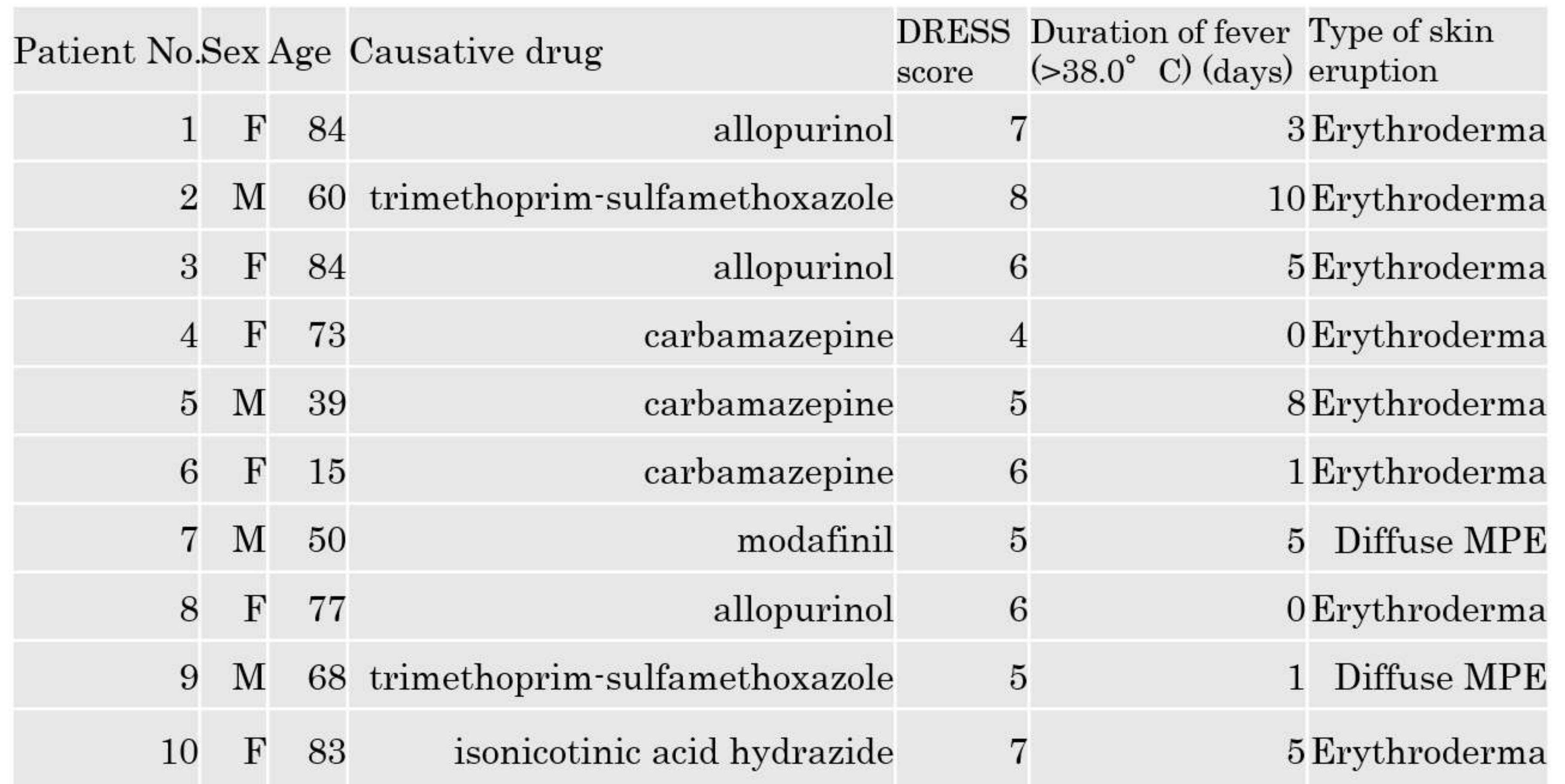

5 Erythroderma

\section{Résūitt}

Up-regulation of HHV-6-derived miRNAs in the serum of DIHS/DRESS patients
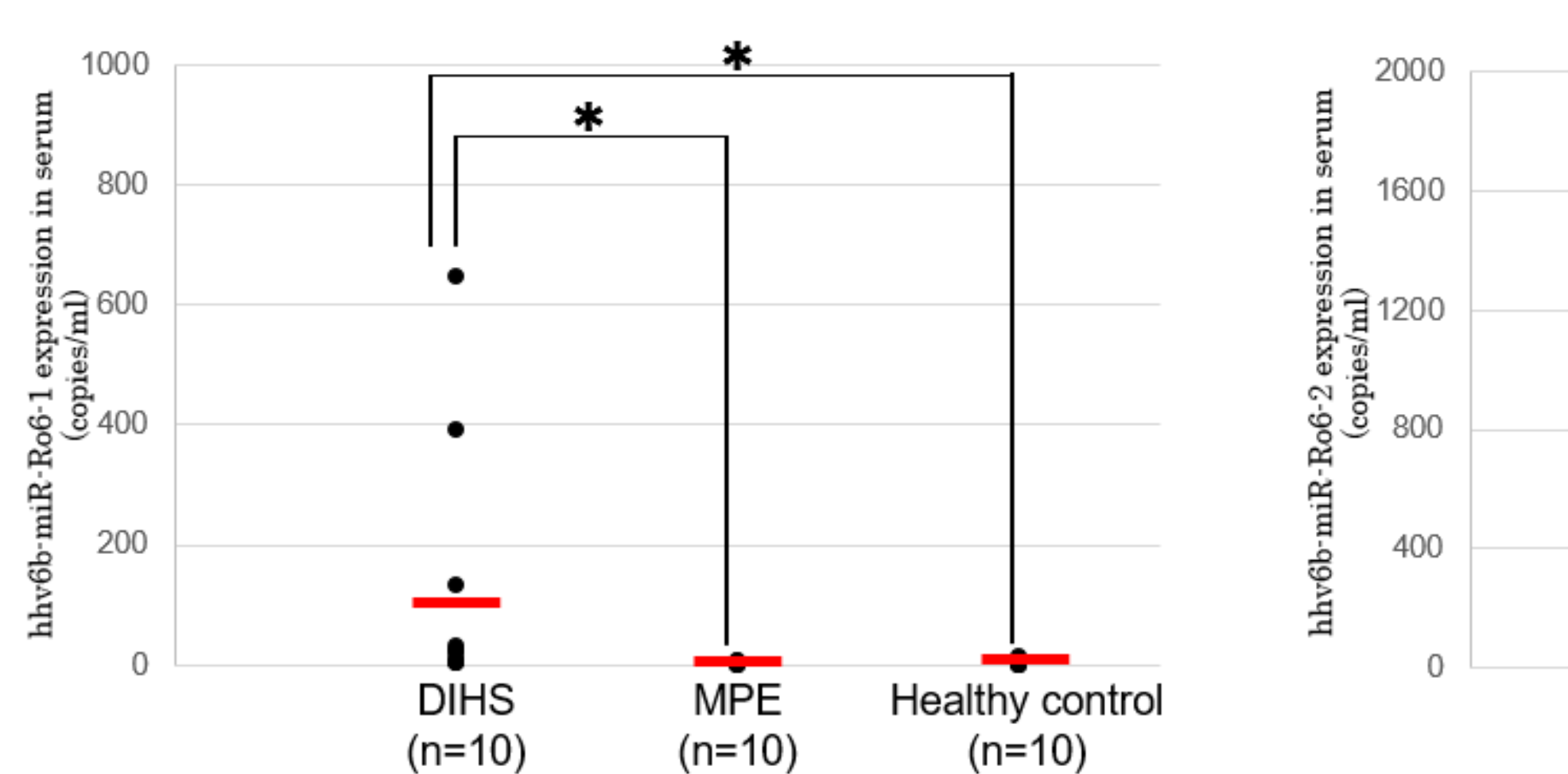

hv6b-miR-Ro6-2

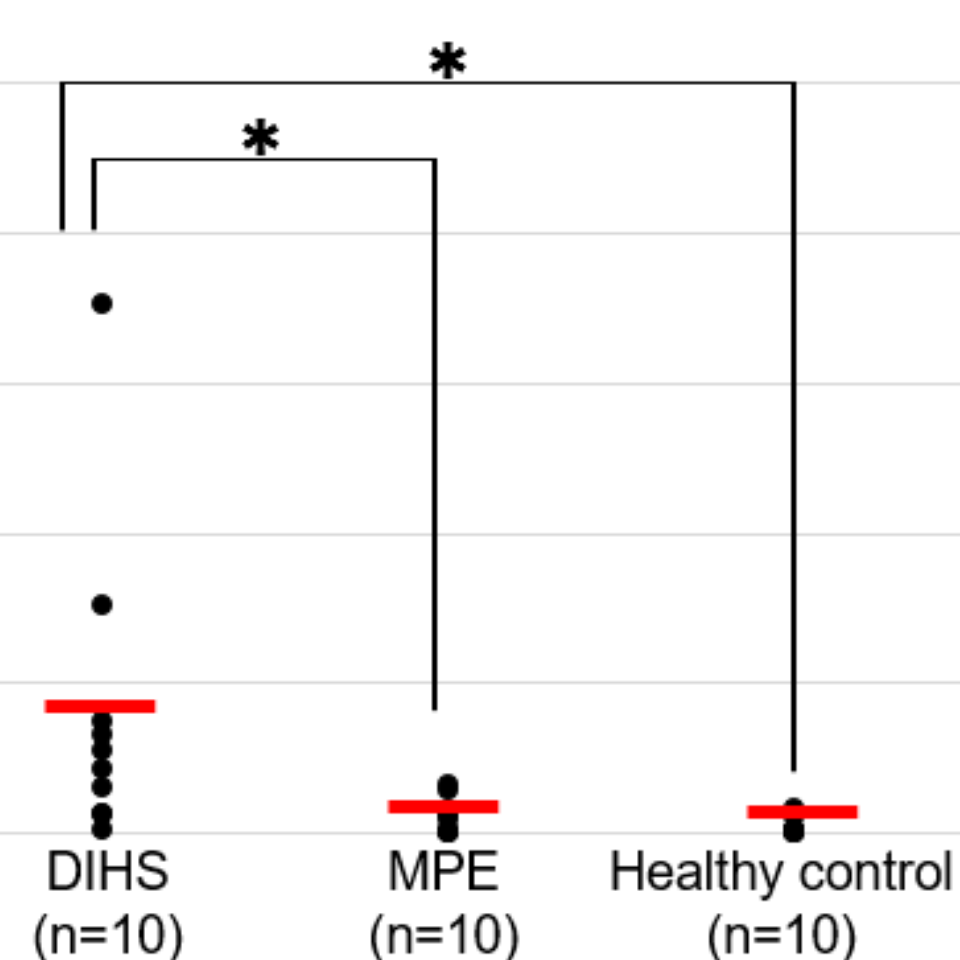

hhv6b-miR-Ro6-3
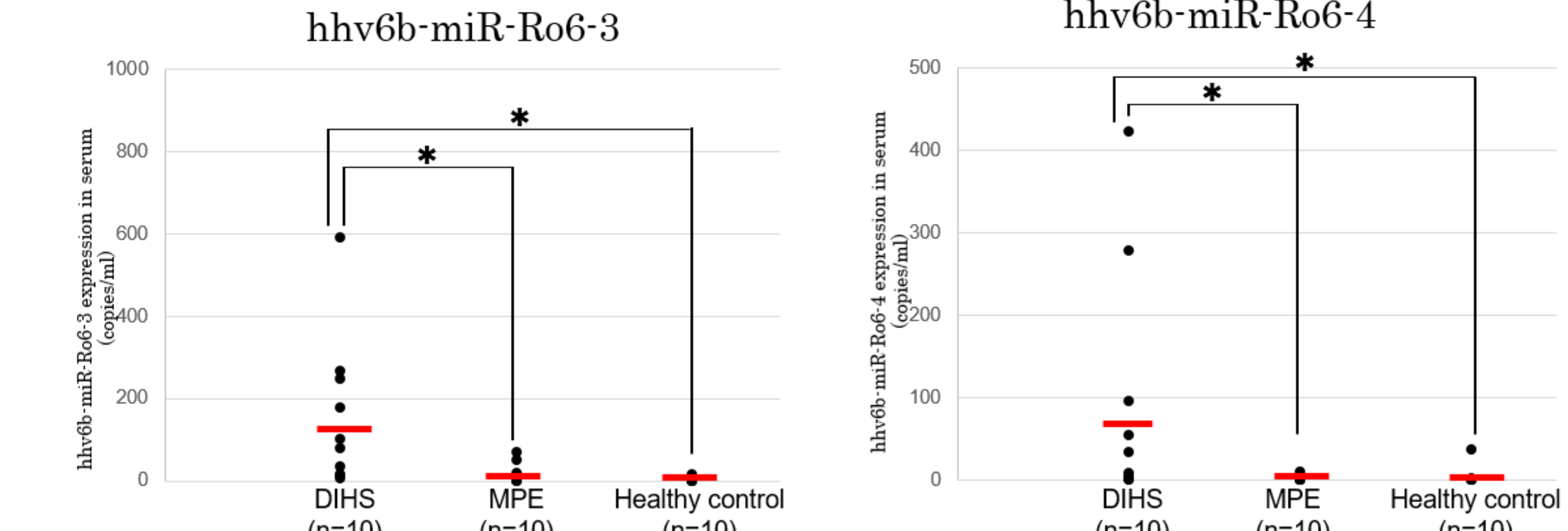

The maximum levels of hhv6b-miR-Ro6-1, $-2,-3$, and -4 in serum were significantly higher in DIHS/DRESS than in MPE patients and healthy controls. ${ }^{*} p<0.05$.

Time course of HHV-6 miRNA expressions in each patient with DIHS/DRESS

(a) Case 1
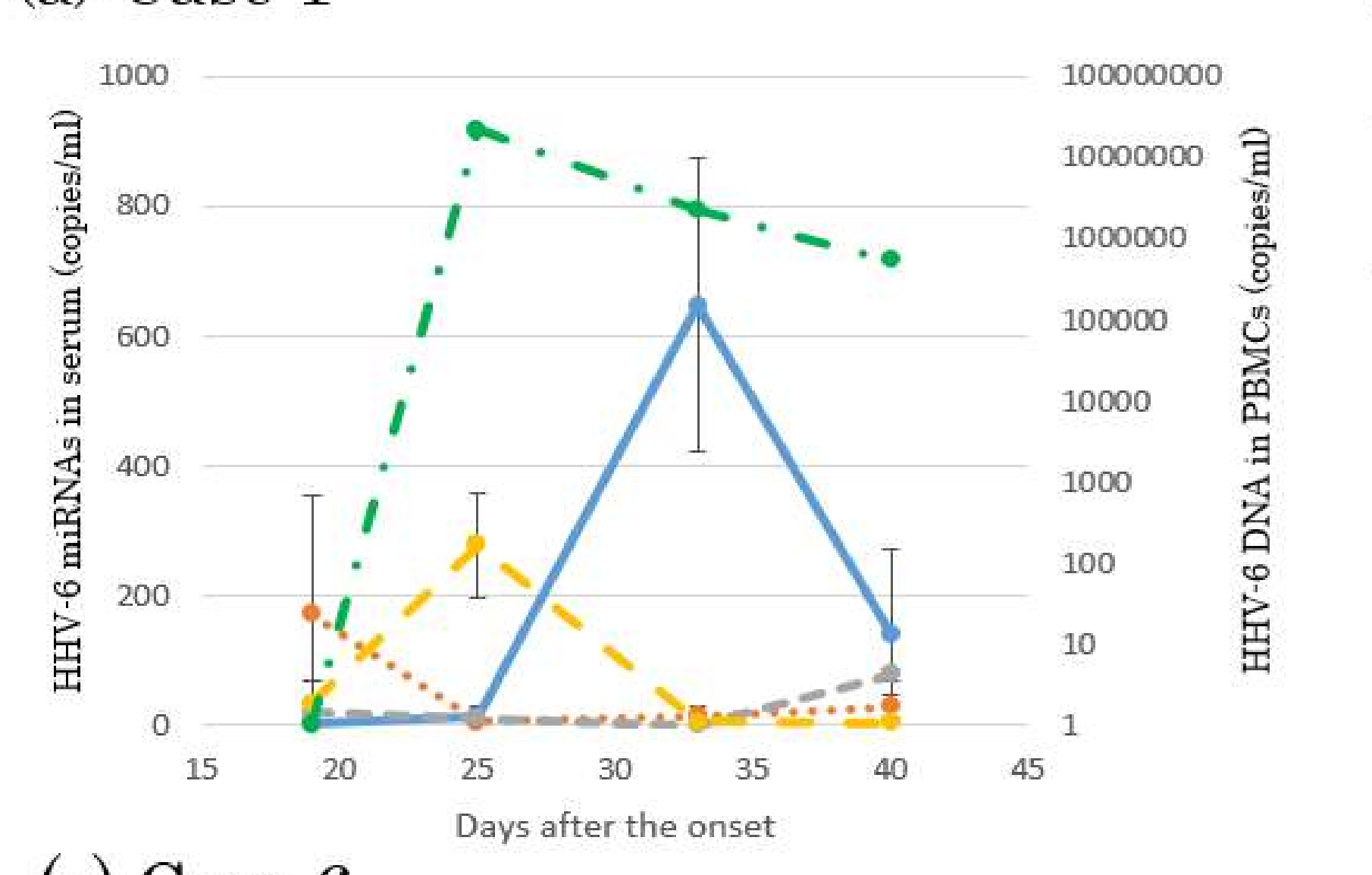

b) Case 2

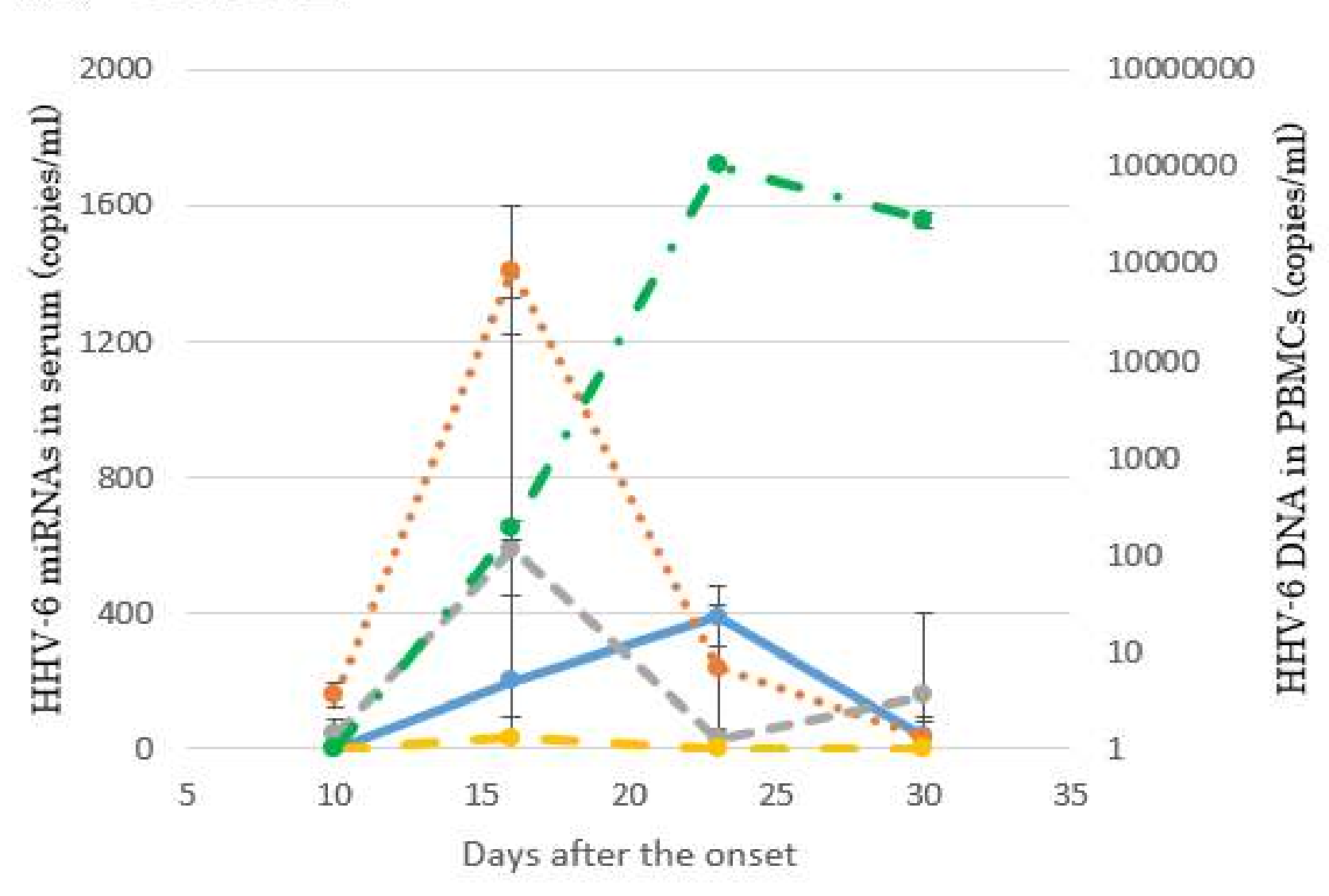

(c) Case 6
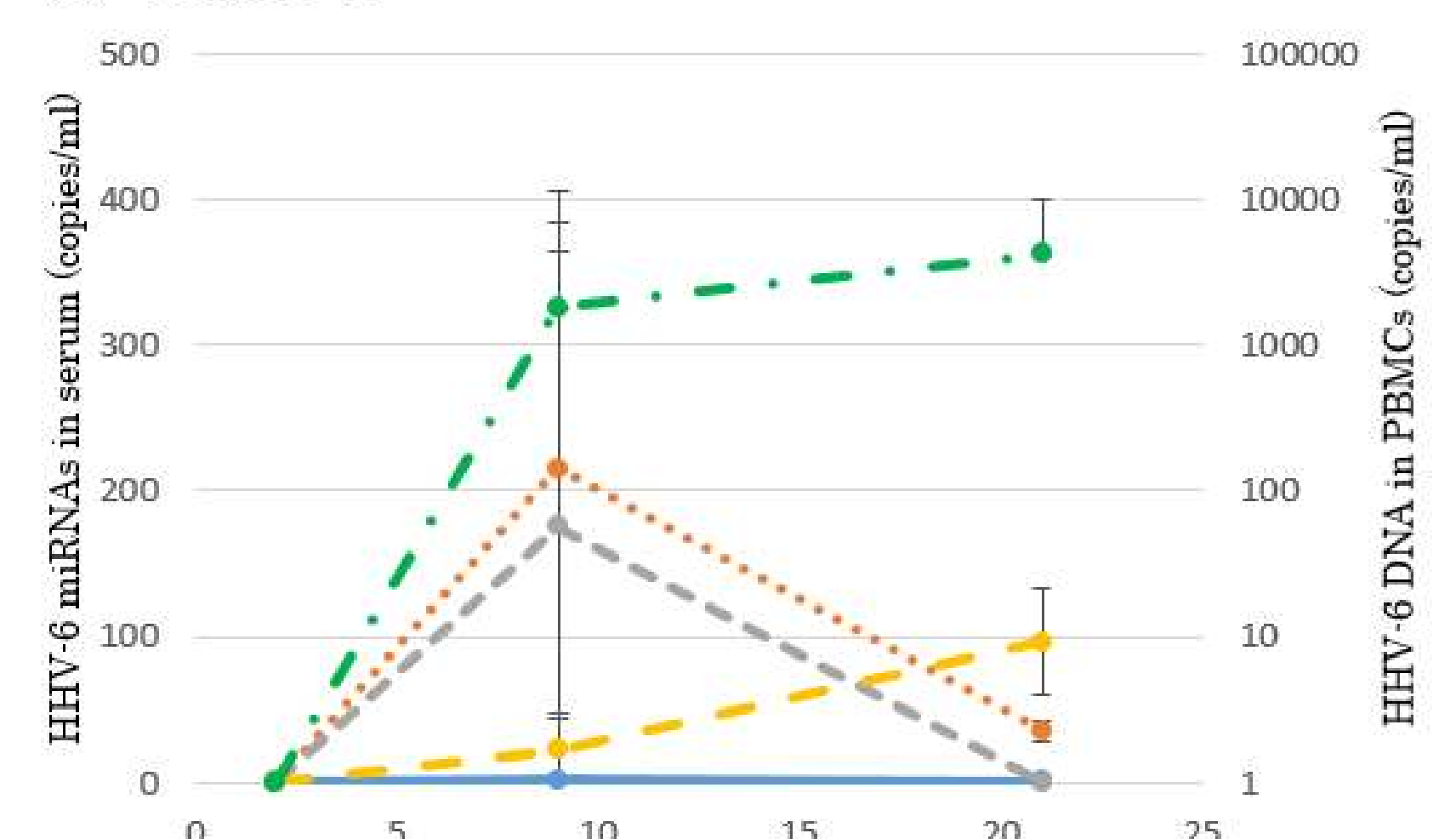

.... hhv6b-miR-Ro6-2 in serum

hhv6b-miR-Ro6-4 in seru

(a) Case1. HHV-6 reactivation was confirmed by detecting HHV-6 DNA in PBMCs on day 25 after onset. The expression of hhv6b-miR-Ro6-2 in serum was detected on day 19, while hhv6b-miR-Ro6-4 and -1 were detected on days 25 and 33 , respectively

(b) Case 2. HHV-6 reactivated on day 16 after onset. Hhv6b-miR-Ro6-2 was expressed on day 10, while hhv6b-miR-Ro6-3 and -1 were expressed on the same day when HHV-6 DNA was detected.

(c) Case 6. The expression of HHV-6 DNA and hhv6b-miR-Ro6-2 and -3 was detectable on day 9 afte onset, while hhv6b-miR-Ro6-4 was detected on day 21 following hhv6b-miR-Ro6-2 expression

\section{Correlation between HHV-6 miRNAs and type of skin eruptior}

\begin{tabular}{|c|c|c|c|c|c|c|}
\hline $\begin{array}{l}\text { Patient No. hhv6b- } \\
\text { Ro6-1 }\end{array}$ & $\begin{array}{l}\text { Serum } \\
\text { miR- }\end{array}$ & $\begin{array}{l}\text { levels of HHV- } \\
\text { hhv66-miR- } \\
\text { Ro6-2 }\end{array}$ & $\begin{array}{l}-6 \text { mikNAs cor } \\
\text { hhv6-miR- } \\
\text { Ro } 0-3\end{array}$ & $\begin{array}{l}\text { opiessmi) } \\
\text { hhv6-miR- } \\
\text { Ro6-4 }\end{array}$ & $\begin{array}{l}\text { Type of skin } \\
\text { eruption }\end{array}$ & When the expression levels of hhv6b- \\
\hline 2 & 389.6 & 1409.3 & $3 \quad 590.3$ & $3 \quad 34.0$ & Erythroderma & \\
\hline 10 & 2.3 & 608.1 & 100.1 & 7.9 & Erythroderma & \\
\hline 3 & 131.1 & 297.5 & 246.1 & 423.2 & Erythroderma & fi \\
\hline 5 & 20.6 & 258.5 & 175.2 & 1.0 & Erythroderma & 206-2 had erythroderma, \\
\hline 6 & 1.9 & 215.9 & 17.5 & 96.3 & Erythroderma & while the last two patients with lower \\
\hline 1 & 646.4 & 172.0 & 79.0 & 277.9 & Erythroderma & levels of hhv6b-miR-Ro6-2 had diffuse \\
\hline 8 & 31.7 & $122.7 \mathrm{r}$ & 10.6 & 4.0 & Erythroderma & MPE. Hhv6b-miR-Ro6-2 may reflect \\
\hline 4 & 7.9 & 50.9 & 32.4 & 54.7 & Erythroderma & the type of skin eruption. \\
\hline 9 & 1.1 & 47.0 & 6.2 & 0.1 & Diffuse MPE & \\
\hline 7 & 24.0 & $3.7 \mathrm{~F}$ & 266.4 & 2.2 & Diffuse MPE & \\
\hline
\end{tabular}

Correlation between DRESS scores and HHV-6 miRNAs in the serum of DIHS/DRESS patients

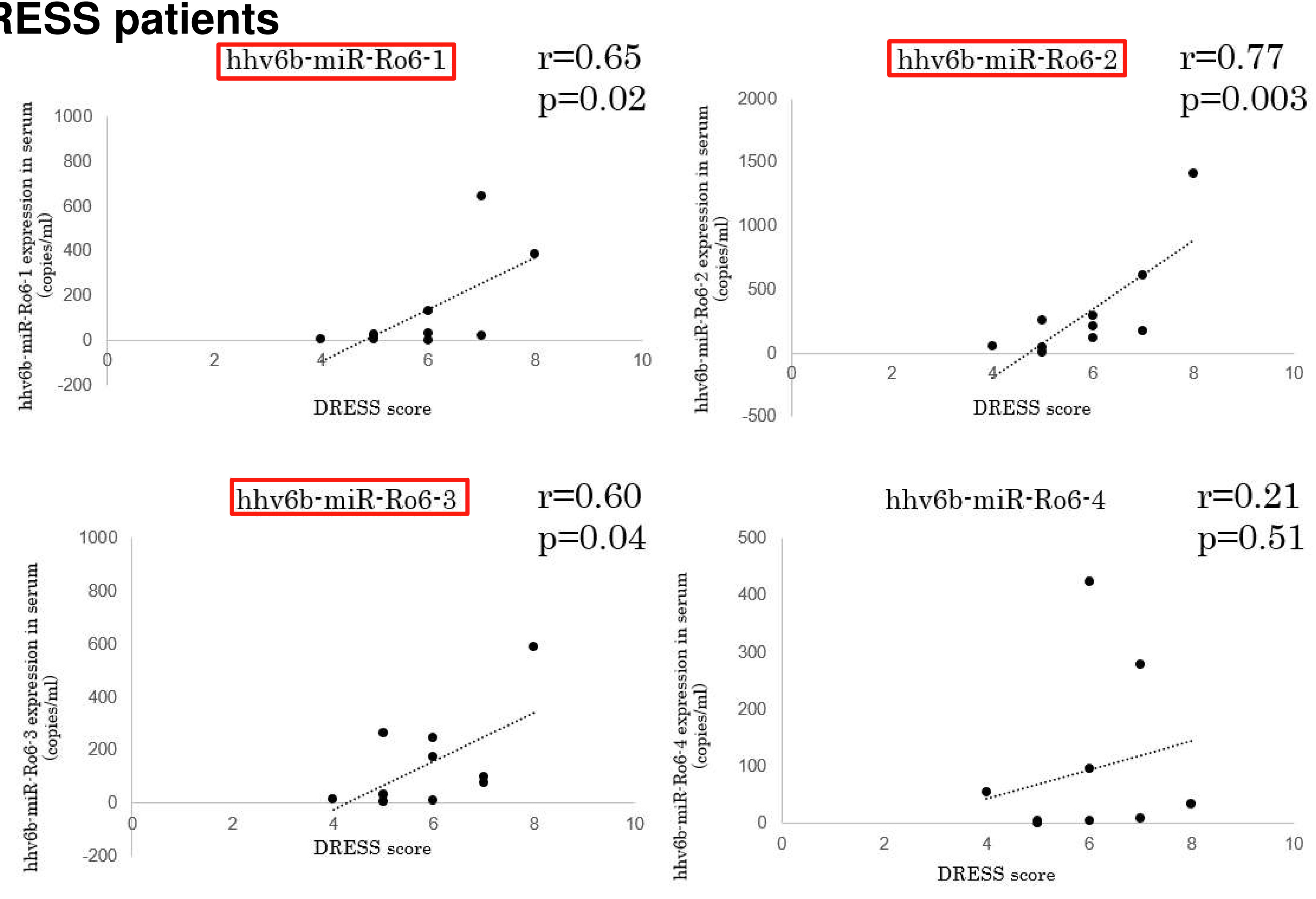

DRESS scores correlated with the serum levels of hhv6b-miR-Ro6-1, -2 , and -3 , respectively.

Correlation between the duration of fever $\left(>38^{\circ} \mathrm{C}\right)$ and HHV-6 miRNAs in the serum of DIHS/DRESS patients.
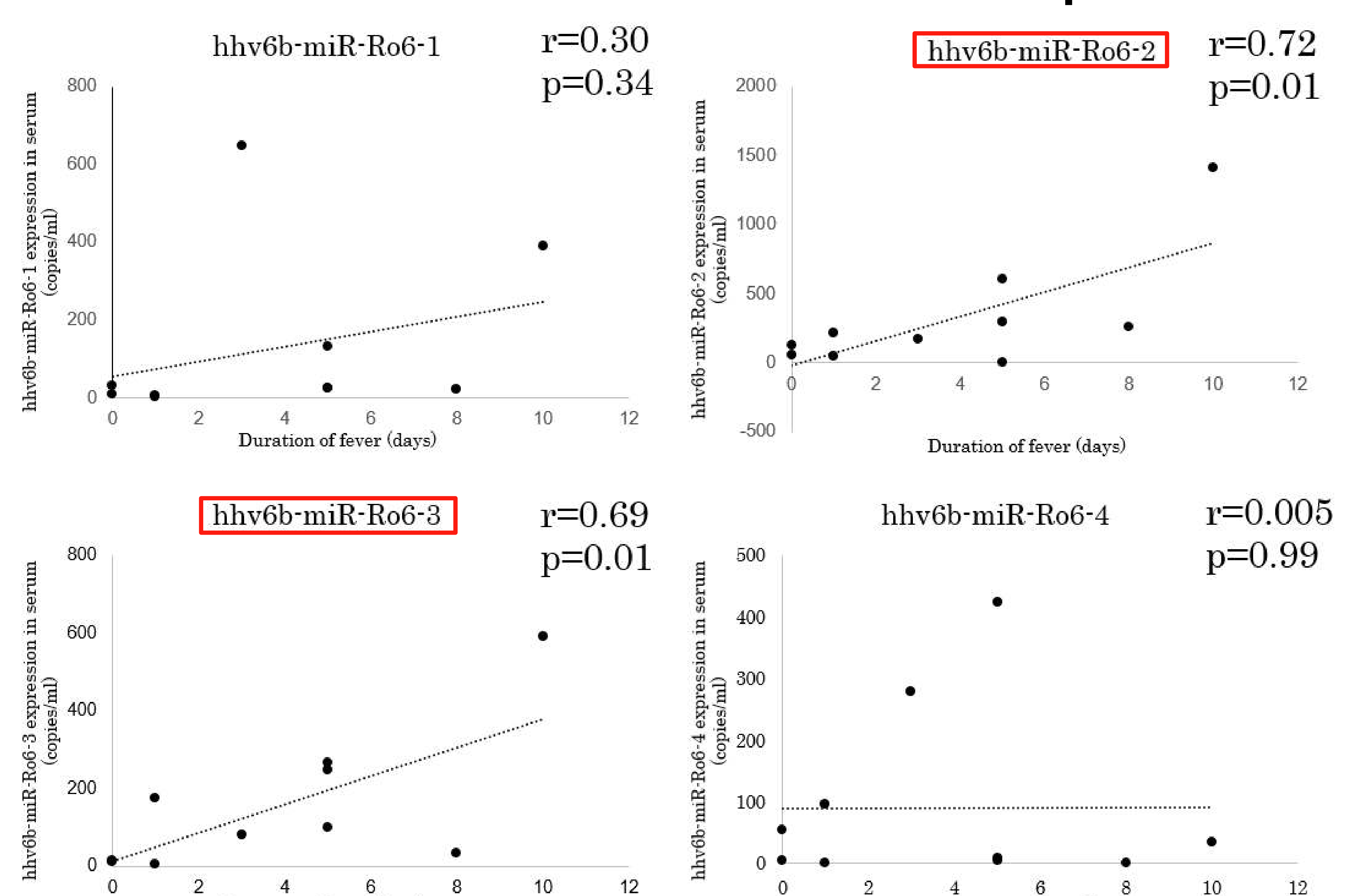

The duration of fever correlated with serum levels of hhv6b-miR-Ro6-2 and -3 , respectively.

\section{Summary}

- Four HHV-6 miRNAs were detected in some DIHS/DRESS patients, but not in MPE patients and healthy controls.

- Serum levels of hhv6b-miR-Ro6-2 increased before or at the same time as the detection of HHV-6 DNA, while serum levels of hhv6b-miR-Ro6-1 and/or 4 significantly increased a few weeks later in some patients with DIHS/DRESS.

- Some HHV-6 miRNAs in the serum are useful indicators of the severity of

DIHS/DRESS.

- Hhv6b-miR-Ro6-2 may be an early and specific biomarker for predicting the reactivation of HHV-6.

- The detection of the miRNAs of HHV-6 in DIHS/DRESS may reflect the reactivation of HHV-6. 